\title{
Commutation Formulae With Respect to Non-Symmetric Affine Connection
}

\author{
Nenad O. Vesić; Dušan J. Simjanović
}

\begin{abstract}
Commutation formulae with respect to a non-symmetric affine connection are obtained in this paper. The components of commutation formulae in this paper are covariant derivatives of tensors with respect to symmetric and non-symmetric affine connection.
\end{abstract}

Key words: covariant derivative, commutation formula, linear independence

2010 Math. Subj. Classification: 53B05, 15A03

\section{Introduction}

Identities of Ricci Type [2 13, 15] are important for different researches in the fields of differential geometry and the corresponding applications.

One curvature tensor of a symmetric affine connection space is obtained with respect to a symmetric affine connection [2,12]. Many curvature tensors and curvature pseudotensors are founded with respect to a non-symmetric affine connection [3, 11, 13, 15]. Curvature tensors and curvature pseudotensors are components of the curvature for the corresponding affine connection spaces.

Our purpose is to obtain all identities of Ricci Type with respect to a non-symmetric affine connection in this paper. In this research, we will try to simplify the previously obtained identities.

\subsection{Affine connection space}

An $N$-dimensional manifold $\mathcal{M}_{N}$ equipped with an affine connection with torsion $\nabla$ is the generalized affine connection space $\mathbb{G A}_{N}$ (see [1, 3, 11, 13, 15]).

The affine connection coefficients with respect to the affine connection (with torsion) $\nabla$ are $L_{j k}^{i}, L_{j k}^{i} \neq L_{k j}^{i}$. The symmetric and anti-symmetric parts of the affine connection coefficients $L_{j k}^{i}$ are

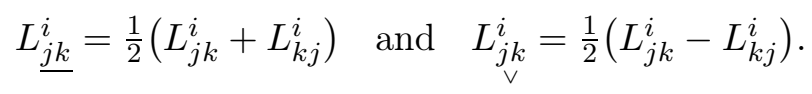

\footnotetext{
${ }^{*}$ This work was supported by the Serbian Ministry of Education, Science and Technological Development through Mathematical Institute of the Serbian Academy of Sciences and Arts
} 
The double anti-symmetric parts $L_{\substack{j k \\ i}}$ are the components of the torsion tensor for the affine connection space $\mathbb{G A}_{N}$.

The symmetric parts $L_{\underline{j k}}^{i}$ satisfy the transformation rule

$$
L_{\underline{j^{\prime} k^{\prime}}}^{i^{\prime}}=x_{i}^{i^{\prime}} x_{j^{\prime}}^{j} x_{k^{\prime}}^{k} L_{\underline{j k}}^{i}+x_{i}^{i^{\prime}} x_{j^{\prime} k^{\prime}}^{i}
$$

For this reason, the manifold $\mathcal{M}_{N}$ equipped with the symmetric affine connection $\stackrel{0}{\nabla}$ whose coefficients are $L_{\underline{j k}}^{i}$ is the associated (symmetric affine connection) space $\mathbb{A}_{N}$ of the space $\mathbb{G A}_{N}$ (see [2,12]).

Covariant derivatives are defined with respect to torsion-free affine connections [2, 12] and affine connections with torsion [1,3, 11, 13, 15, With respect to double covariant derivatives, corresponding commutation formulae are obtained. From the commutation formulae, the curvature tensors for the spaces $\mathbb{A}_{N}$ and $\mathbb{G}_{N}$ are founded.

\subsection{About covariant derivatives}

It exists one kind of covariant derivative with respect to the affine connection $\stackrel{0}{\nabla}$ (see [2, 12])

$$
a_{j_{1} \ldots j_{q} \mid k}^{i_{1} \ldots i_{p}}=a_{j_{1} \ldots j_{q}, k}^{i_{1} \ldots i_{p}}+\sum_{u=1}^{p} L_{\underline{\alpha k}}^{i_{u}} a_{j_{1} \ldots j_{q}}^{i_{1} \ldots i_{u-1} \alpha i_{u+1} \ldots i_{p}}-\sum_{v=1}^{q} L_{\underline{j_{v} k}}^{\alpha} a_{j_{1} \ldots j_{v-1} \alpha j_{v+1} \ldots j_{q}}^{i_{1} \ldots i_{p}},
$$

for a tensor $\hat{a}$ of the type $(p, q)$ whose components are $a_{j_{1} \ldots j_{q}}^{i_{1} \ldots i_{p}}$ and the partial derivative $\partial / \partial x^{k}$ denoted by comma.

It exists one Ricci-Type identity with respect to the covariant derivative given by the equation $(1.3)$

$$
a_{j_{1} \ldots j_{q}|m| n}^{i_{1} \ldots i_{p}}-a_{j_{1} \ldots j_{q}|n| m}^{i_{1} \ldots i_{p}}=\sum_{u=1}^{p} a_{j_{1} \ldots j_{q}}^{i_{1} \ldots i_{u-1} \alpha i_{u+1} \ldots i_{p}} R_{\alpha m n}^{i}-\sum_{v=1}^{q} a_{j_{1} \ldots j_{v-1} \alpha j_{v+1} \ldots j_{q}}^{i_{1} \ldots i_{p}} R_{j_{v} m n}^{\alpha}
$$

for the components

$$
R_{j m n}^{i}=L_{\underline{j m}, n}^{i}-L_{\underline{j n}, m}^{i}+L_{\underline{j m}}^{\alpha} L_{\underline{\alpha n}}^{i}-L_{\underline{j n}}^{\alpha} L_{\underline{\alpha m}}^{i},
$$

of the curvature tensor $\hat{R}$ of the type $(1,3)$ for the associated space $\mathbb{A}_{N}$.

There are four kinds of covariant derivatives with respect to the affine connection with torsion $\nabla($ see $[1,3,11,13,15])$ 


$$
\begin{aligned}
& a_{j_{1} \ldots j_{q} \mid k}^{i_{1} \ldots i_{p}}=a_{j_{1} \ldots j_{q}, k}^{i_{1} \ldots i_{p}}+\sum_{u=1}^{p} L_{\alpha k}^{i_{u}} a_{j_{1} \ldots j_{q}}^{i_{1} \ldots i_{u-1} \alpha i_{u+1} \ldots i_{p}}-\sum_{v=1}^{q} L_{j_{v} k}^{\alpha} a_{j_{1} \ldots j_{v-1} \alpha j_{v+1} \ldots j_{q}}^{i_{1} \ldots i_{p}}, \\
& a_{j_{1} \ldots j_{q} \mid k}^{i_{1} \ldots i_{p}}=a_{j_{1} \ldots j_{q}, k}^{i_{1} \ldots i_{p}}+\sum_{u=1}^{p} L_{k \alpha}^{i_{u}} a_{j_{1} \ldots j_{q}}^{i_{1} \ldots i_{u-1} \alpha i_{u+1} \ldots i_{p}}-\sum_{v=1}^{q} L_{k j_{v}}^{\alpha} a_{j_{1} \ldots j_{v-1} \alpha j_{v+1} \ldots j_{q}}^{i_{1} \ldots i_{p}}, \\
& a_{j_{1} \ldots j_{q} \mid k}^{i_{1} \ldots i_{p}}=a_{j_{1} \ldots j_{q}, k}^{i_{1} \ldots i_{p}}+\sum_{u=1}^{p} L_{\alpha k}^{i_{u}} a_{j_{1} \ldots j_{q}}^{i_{1} \ldots i_{u-1} \alpha i_{u+1} \ldots i_{p}}-\sum_{v=1}^{q} L_{k j_{v}}^{\alpha} a_{j_{1} \ldots j_{v-1} \alpha j_{v+1} \ldots j_{q}}^{i_{1} \ldots i_{p}}, \\
& a_{j_{1} \ldots j_{q} \mid k}^{i_{1} \ldots i_{p}}=a_{j_{1} \ldots j_{q}, k}^{i_{1} \ldots i_{p}}+\sum_{u=1}^{p} L_{k \alpha}^{i_{u}} a_{j_{1} \ldots j_{q}}^{i_{1} \ldots i_{u-1} \alpha i_{u+1} \ldots i_{p}}-\sum_{v=1}^{q} L_{j_{v} k}^{\alpha} a_{j_{1} \ldots j_{v-1} \alpha j_{v+1} \ldots j_{q}}^{i_{1} \ldots i_{p}}
\end{aligned}
$$

Let be $a_{j_{1} \ldots j_{q} \mid k}^{i_{1} \ldots i_{p}} \equiv a_{j_{1} \ldots j_{q} \mid k}^{i_{1} \ldots i_{p}}$. We will study the differences $a_{j_{1} \ldots j_{q}|m| n}^{i_{v_{1}} \ldots i_{w_{1}}}-a_{j_{1} \ldots j_{q}|n| m}$, $v_{1}, v_{2}, w_{1}, w_{2} \in\{0,1,2,3,4\}$, in this paper.

\subsection{Motivation}

It is obtained the Ricci-Type identity [1, 3, 11, 13, 15]

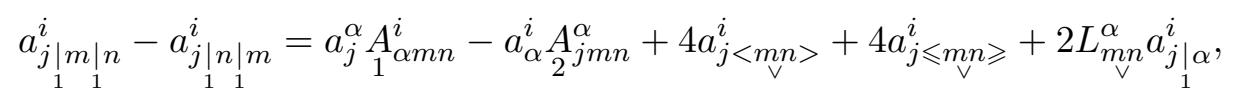

for

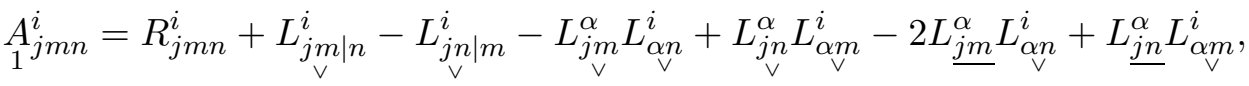

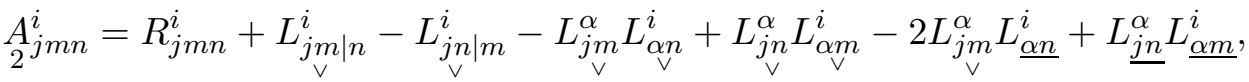

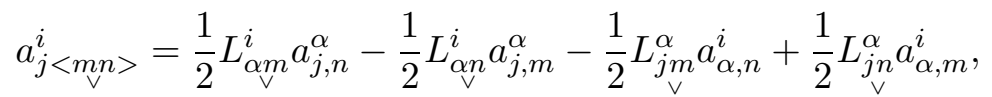

$$
\begin{aligned}
& a_{j \leqslant m n}^{i} \geqslant=\frac{1}{2} a_{\beta}^{\alpha}\left(L_{m \beta}^{i} L_{j n}^{\alpha}-L_{n \beta}^{i} L_{j m}^{\alpha}-L_{\alpha m}^{i} L_{n j}^{\beta}+L_{\alpha n}^{i} L_{m j}^{\beta}\right) \text {. }
\end{aligned}
$$

The geometrical objects $\underset{1}{A_{j m n}^{i}}$ and $\underset{2}{A_{j m n}}$ are components of the curvature pseudotensors $\hat{A}$ and $\underset{2}{\hat{A}}$ of the type $(1,3)$. These objects are components of the curvature for the space $\mathbb{G}_{N}$.

In [14], and with respect to $L_{j k}^{i}=L_{\underline{j k}}^{i}+\underset{\substack{j k \\ \text {, the equation (1.10) }}}{i}$ is simplified to

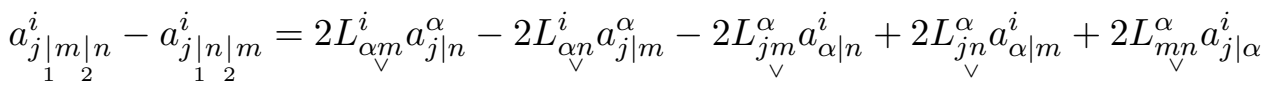

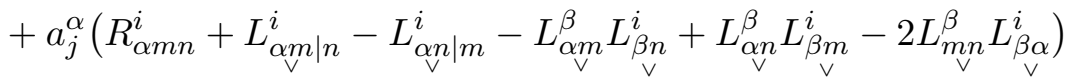

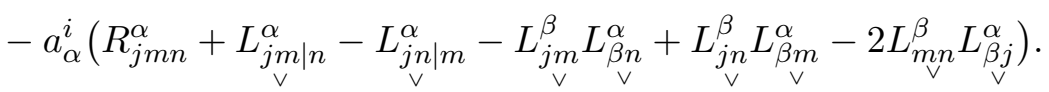

In [14], it is obtained the family of double covariant derivatives 


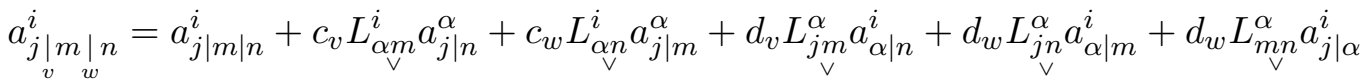

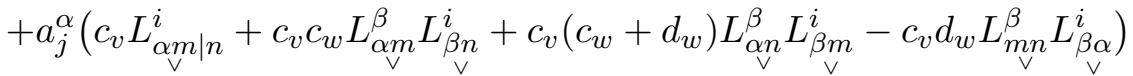

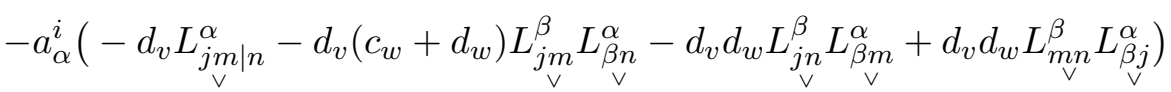

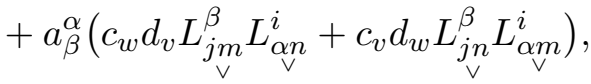

for $v, w \in\{0,1,2,3,4\}$.

When simplified the difference $a_{j|m| v_{1} \mid w_{1}}^{i}-a_{j_{v_{2} \mid w_{2}}}^{i}$, we proved the next theorem.

Theorem 1.1 (First Ricci-Type Identities Theorem). [14] The family of identities of the Ricci Type with respect to a non-symmetric affine connection $\nabla$ is

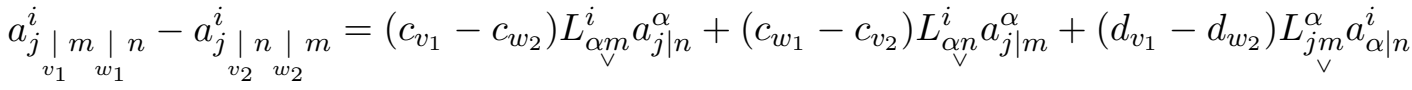

$$
\begin{aligned}
& +\left(d_{w_{1}}-d_{v_{2}}\right) L_{j n}^{\alpha} a_{\alpha \mid m}^{i}+\left(d_{w_{1}}+d_{w_{2}}\right) L_{\underset{v}{ } n}^{\alpha} a_{j \mid \alpha}^{i} \\
& +a_{j}^{\alpha}\left\{R_{\alpha m n}^{i}+c_{v_{1}} L_{\alpha m \mid n}^{i}-c_{v_{2}} L_{\alpha n \mid m}^{i}\right. \\
& +\left[c_{v_{1}} c_{w_{1}}-c_{v_{2}}\left(c_{w_{2}}+d_{w_{2}}\right)\right] L_{\underset{\vee}{ }}^{\beta} L_{\beta n}^{i} \\
& +\left[c_{v_{1}}\left(c_{w_{1}}+d_{w_{1}}\right)-c_{v_{2}} c_{w_{2}}\right] L_{\vee \sim}^{\beta} L_{\beta m}^{i} \\
& \left.-\left(c_{v_{1}} d_{w_{1}}+c_{v_{2}} d_{w_{2}}\right) L_{\underset{\vee}{m}}^{\beta} L_{\beta \alpha}^{i}\right\}
\end{aligned}
$$

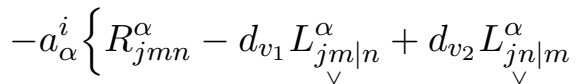

$$
\begin{aligned}
& -\left[d_{v_{1}}\left(c_{w_{1}}+d_{w_{1}}\right)-d_{v_{2}} d_{w_{2}}\right] L_{j \mathrm{v}}^{\beta} L_{\beta n}^{\alpha} \\
& -\left[d_{v_{1}} d_{w_{1}}-d_{v_{2}}\left(c_{w_{2}}+d_{w_{2}}\right)\right] \underset{\vee}{L_{\vee n}} L_{\beta m}^{\alpha}
\end{aligned}
$$

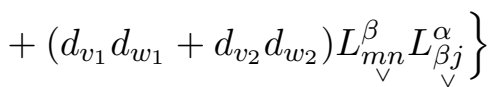

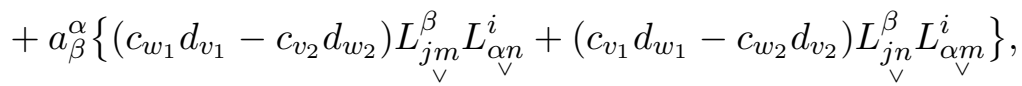

for $v_{1}, v_{2}, w_{1}, w_{2} \in\{0,1,2,3,4\}$.

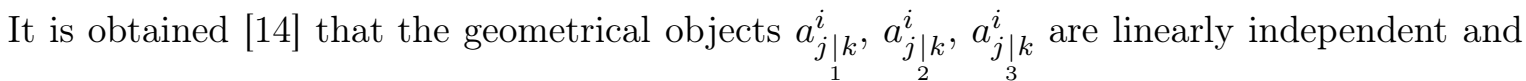
that the geometrical objects $a_{j \mid k}^{i}$ and $a_{j \mid k}^{i}$ may be uniquely expressed in the terms of the first three kinds of covariant derivative.

The purpose of this paper is to generalize the First Ricci-Type Identities Theorem in the sense of changing the summands $L_{j k}^{i} a_{s \mid r}^{l}$ with linear combinations of the geometrical objects

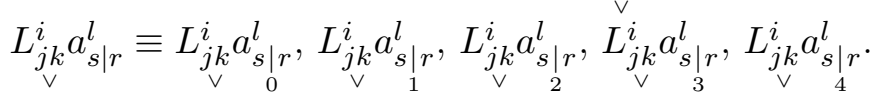


At the start of the research, we will prove that three of covariant derivatives $a_{j \mid k}^{i}, a_{\substack{1 \\ i}}^{i}, a_{j \mid k}^{i}$, $a_{j \mid k}^{i}, a_{j \mid k}^{i}$ are enough for all commutation formulae to be obtained.

The next result of our research will be the commutation formulae with respect to double covariant derivatives of a tensor $\hat{a}$ of a type $(p, q), p, q \in \mathbb{N}$.

\section{Four plus one kinds of covariant derivatives}

For the research in this paper, we need the next propositions.

Proposition 2.1. The covariant derivatives given by the equations (1.6, 1.7, 1.8, 1.9) and the covariant derivative given by the equation (1.3) satisfy the equations

$$
\begin{aligned}
& a_{j_{1} \ldots j_{q} \mid k}^{i_{1} \ldots i_{p}}=a_{j_{1} \ldots j_{q} \mid k}^{i_{1} \ldots i_{p}}+\sum_{u=1}^{p} L_{\alpha \vee}^{i_{u}} a_{j_{1} \ldots j_{q}}^{i_{1} \ldots i_{u-1} \alpha i_{u+1} \ldots i_{p}}-\sum_{v=1}^{q} L_{j_{v} k}^{\alpha} a_{j_{1} \ldots j_{v-1} \alpha j_{v+1} \ldots j_{q}}^{i_{1} \ldots i_{p}}, \\
& a_{j_{1} \ldots j_{q} \mid k}^{i_{1} \ldots i_{p}}=a_{j_{1} \ldots j_{q} \mid k}^{i_{1} \ldots i_{p}}-\sum_{u=1}^{p} L_{\alpha k}^{i_{u}} a_{j_{1} \ldots j_{q}}^{i_{1} \ldots i_{u-1} \alpha i_{u+1} \ldots i_{p}}+\sum_{v=1}^{q} L_{j_{v} k}^{\alpha} a_{j_{1} \ldots j_{v-1} \alpha j_{v+1} \ldots j_{q}}^{i_{1} \ldots i_{p}},
\end{aligned}
$$

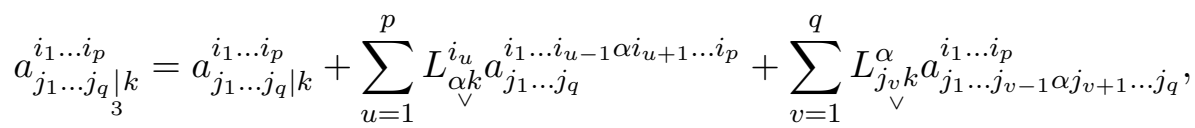

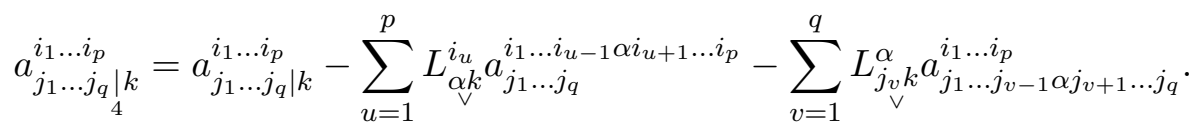

Remark 2.1. With respect to the equations (1.3, 2.1,2.4), we obtain

$$
a_{j_{1} \ldots j_{q} \mid k}^{i_{1} \ldots i_{p}}=a_{j_{1} \ldots j_{q} \mid k}^{i_{1} \ldots i_{p}}+c_{z} \sum_{u=1}^{p} L_{\substack{\alpha \\ i_{u}}}^{i_{j_{1} \ldots j_{q}}} a_{j_{1} \ldots i_{u-1} \alpha i_{u+1} \ldots i_{p}}+d_{z} \sum_{v=1}^{q} L_{j_{v} k}^{\alpha} a_{j_{1} \ldots j_{v-1} \alpha j_{v+1} \ldots j_{q}}^{i_{1} \ldots i_{p}},
$$

for $z=0, \ldots, 4$, and the corresponding coefficients $c_{0}=d_{0}=0, c_{1}=1, c_{2}=-1, c_{3}=1$, $c_{4}=-1, d_{1}=-1, d_{2}=1, d_{3}=1, d_{4}=-1$.

Let us obtain the commutation formulae with respect to covariant derivatives of tensors $\hat{a}$ of the type $(1,1), \hat{u}$ of the type $(1,0)$ and $\hat{v}$ of the type $(0,1)$.

Proposition 2.2. For a tensor $\hat{a}$ of the type $(1,1)$, three of the geometrical objects $a_{\substack{j \mid k \\ i}}, a_{j \mid k}^{i}$,

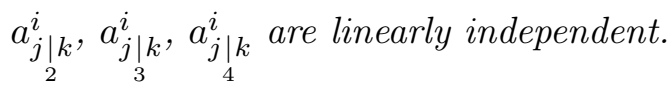

Proof. With respect to the equation (2.5), the number of linearly independent geometrical objects $a_{\substack{j \mid k \\ i}}^{i}, a_{j \mid k}^{i}, a_{\substack{j \mid k \\ j}}^{i}, a_{\substack{\mid k \\ j}}^{i}, a_{j \mid k}^{i}$ is equal to the rank of the matrix

$$
M=\left[\begin{array}{ccc}
1 & 0 & 0 \\
1 & 1 & -1 \\
1 & -1 & 1 \\
1 & 1 & 1 \\
1 & -1 & -1
\end{array}\right]
$$




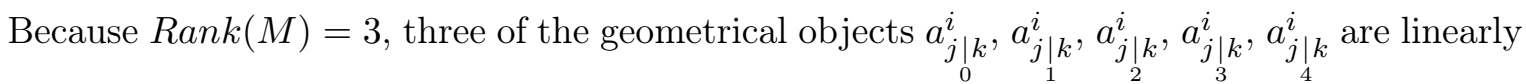
independent.

Corollary 2.1. For a tensor $\hat{u}$ of the type $(1,0)$, two of the geometrical objects $\underset{\substack{\mid k \\ i}}{i}, u_{\substack{\mid k \\ i}}^{i} \equiv \underset{\substack{\mid k k \\ u^{2}}}{i}$,

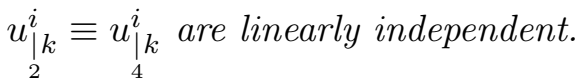

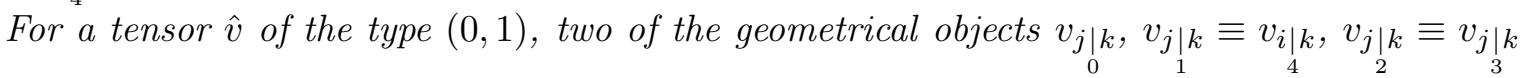
are linearly independent.

Corollary 2.2. The triples

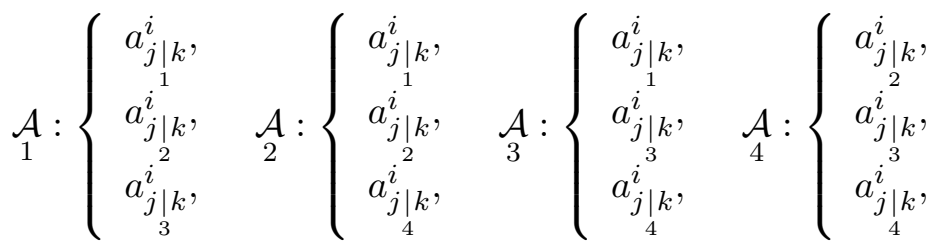

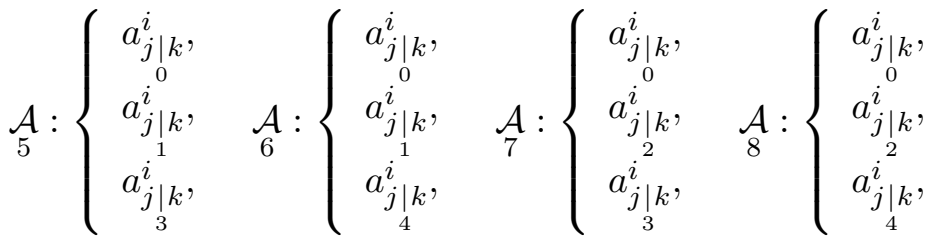

are triples of linearly independent geometrical objects $a_{j \mid k}^{i}, z=0, \ldots, 4$.

The pairs

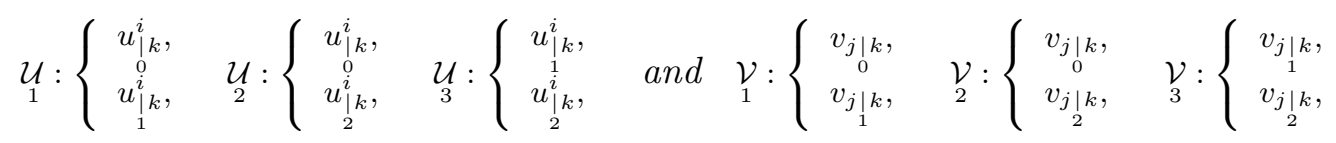

are pairs of linearly independent geometrical objects $\underset{z}{u_{\mid k}^{i}, v_{j} \mid k}$, for $z=0, \ldots, 4$.

\section{Identities of Ricci Type with respect to tensor $\hat{a}$ of type $(1,1)$}

Let us generalize the First Ricci-Type Identities Theorem.

Theorem 3.1 (Second Ricci-Type Identities Theorem). Let be

$$
\begin{aligned}
& X_{j k}^{i}=\rho_{0}^{1} a_{j \mid k}^{i}+\rho_{1}^{1} a_{j \mid k}^{i}+\rho_{2}^{1} a_{j \mid k}^{i}+\rho_{3}^{1} a_{j \mid k}^{i}+\rho_{4}^{1} a_{j \mid k}^{i}, \\
& Y_{j k}^{i}=\rho_{0}^{2} a_{j \mid k}^{i}+\rho_{1}^{2} a_{j \mid k}^{i}+\rho_{2}^{2} a_{j \mid k}^{i}+\rho_{3}^{2} a_{j \mid k}^{i}+\rho_{4}^{2} a_{j \mid k}^{i}, \\
& Z_{j k}^{i}=\rho_{0}^{3} a_{j \mid k}^{i}+\rho_{1}^{3} a_{j \mid k}^{i}+\rho_{2}^{3} a_{j \mid k}^{i}+\rho_{3}^{3} a_{j \mid k}^{i}+\rho_{4}^{3} a_{j \mid k}^{i}, \\
& U_{j k}^{i}=\rho_{0}^{4} a_{j \mid k}^{i}+\rho_{1}^{4} a_{j \mid k}^{i}+\rho_{2}^{4} a_{j \mid k}^{i}+\rho_{3}^{4} A_{j \mid k}^{i}+\rho_{4}^{4} a_{j \mid k}^{i},
\end{aligned}
$$




$$
V_{j k}^{i}=\rho_{0}^{5} a_{j \mid k}^{i}+\rho_{1}^{5} a_{j \mid k}^{i}+\rho_{2}^{5} a_{j \mid k}^{i}+\rho_{3}^{5} a_{j \mid k}^{i}+\rho_{4}^{5} a_{j \mid k}^{i}
$$

for a tensor $\hat{a}$ of the type $(1,1)$ and scalars $\rho_{0}^{z}, \rho_{1}^{z}, \rho_{2}^{z}, \rho_{3}^{z}, \rho_{4}^{z}, z \in\{1, \ldots, 5\}, \rho_{0}^{z}+\rho_{1}^{z}+\rho_{2}^{z}+\rho_{3}^{z}+\rho_{4}^{z}=$ 1.

It holds the equation

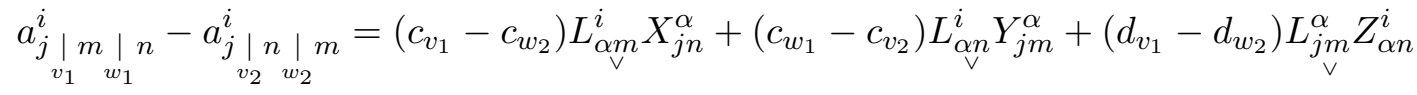

$$
\begin{aligned}
& +\left(d_{w_{1}}-d_{v_{2}}\right) L_{j n}^{\alpha} U_{\alpha m}^{i}+\left(d_{w_{1}}+d_{w_{2}}\right) L_{m_{\vee}}^{\alpha} V_{j \alpha}^{i} \\
& +a_{j}^{\alpha}\left\{R_{\alpha m n}^{i}+c_{v_{1}} L_{\alpha m \mid n}^{i}-c_{v_{2}} L_{\alpha \sqrt{ } \mid m}^{i}\right.
\end{aligned}
$$

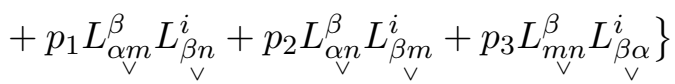

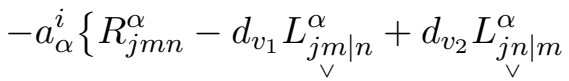

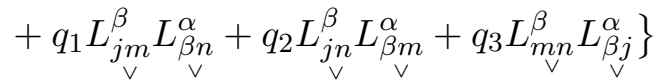

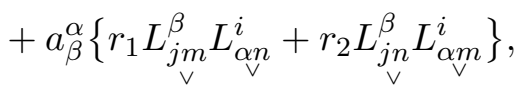

where

$$
\begin{aligned}
p_{1} & =c_{v_{1}} c_{w_{1}}-c_{v_{2}}\left(c_{w_{2}}+d_{w_{2}}\right)-\left(c_{w_{1}}-c_{v_{2}}\right)\left(\rho_{1}^{2}-\rho_{2}^{2}+\rho_{3}^{2}-\rho_{4}^{2}\right), \\
p_{2} & =c_{v_{1}}\left(c_{w_{1}}+d_{w_{1}}\right)-c_{v_{2}} c_{w_{2}}-\left(c_{v_{1}}-c_{w_{2}}\right)\left(\rho_{1}^{1}-\rho_{2}^{1}+\rho_{3}^{1}-\rho_{4}^{1}\right), \\
p_{3} & =-c_{v_{1}} d_{w_{1}}-c_{v_{2}} d_{w_{2}}+\left(d_{w_{1}}+d_{w_{2}}\right)\left(\rho_{1}^{5}-\rho_{2}^{5}+\rho_{3}^{5}-\rho_{4}^{5}\right), \\
q_{1} & =-d_{v_{1}}\left(c_{w_{1}}+d_{w_{1}}\right)+d_{v_{2}} d_{w_{2}}-\left(d_{v_{1}}-d_{w_{2}}\right)\left(\rho_{1}^{3}-\rho_{2}^{3}-\rho_{3}^{3}+\rho_{4}^{3}\right), \\
q_{2} & =-d_{v_{1}} d_{w_{1}}+d_{v_{2}}\left(c_{w_{2}}+d_{w_{2}}\right)-\left(d_{w_{1}}-d_{v_{2}}\right)\left(\rho_{1}^{4}-\rho_{2}^{4}-\rho_{3}^{4}+\rho_{4}^{4}\right), \\
q_{3} & =d_{v_{1}} d_{w_{1}}+d_{v_{2}} d_{w_{2}}-\left(d_{w_{1}}+d_{w_{2}}\right)\left(\rho_{1}^{5}-\rho_{2}^{5}-\rho_{3}^{5}+\rho_{4}^{5}\right), \\
r_{1} & =c_{w_{1}} d_{v_{1}}-c_{v_{2}} d_{w_{2}}+\left(c_{w_{1}}-c_{v_{2}}\right)\left(\rho_{1}^{2}-\rho_{2}^{2}-\rho_{3}^{2}+\rho_{4}^{2}\right) \\
& -\left(d_{v_{1}}-d_{w_{2}}\right)\left(\rho_{1}^{3}-\rho_{2}^{3}+\rho_{3}^{3}-\rho_{4}^{3}\right), \\
r_{2} & =c_{v_{1}} d_{w_{1}}-c_{w_{2}} d_{v_{2}}+\left(c_{v_{1}}-c_{w_{2}}\right)\left(\rho_{1}^{1}-\rho_{2}^{1}-\rho_{3}^{1}+\rho_{4}^{1}\right) \\
& -\left(d_{w_{1}}-d_{v_{2}}\right)\left(\rho_{1}^{4}-\rho_{2}^{4}+\rho_{3}^{4}-\rho_{4}^{4}\right) .
\end{aligned}
$$

Proof. We get

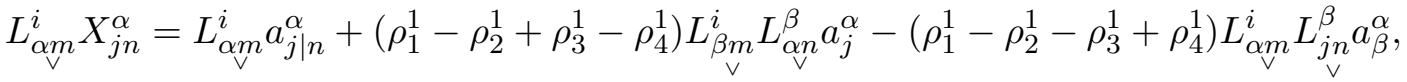

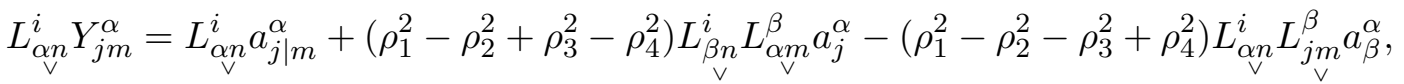

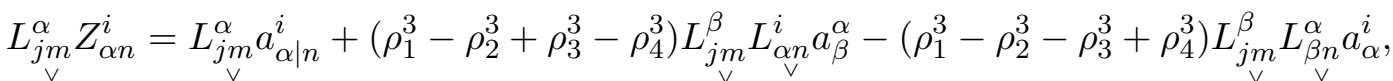

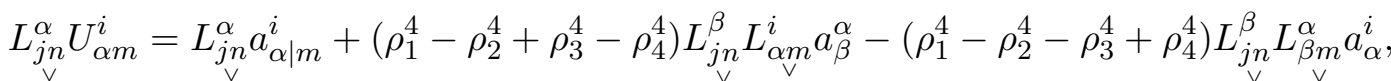

$$
\begin{aligned}
& L_{\underset{\vee}{m} n}^{\alpha} V_{j \alpha}^{i}=L_{m_{\vee} n}^{\alpha} a_{j \mid \alpha}^{i}+\left(\rho_{1}^{5}-\rho_{2}^{5}+\rho_{3}^{5}-\rho_{4}^{5}\right) L_{\underset{\vee}{ } n}^{\beta} L_{\alpha \beta}^{i} a_{j}^{\alpha}-\left(\rho_{1}^{5}-\rho_{2}^{5}-\rho_{3}^{5}+\rho_{4}^{5}\right) L_{\underset{\vee}{ }{ }^{\beta}}^{\beta} L_{\beta j}^{\alpha} a_{\alpha}^{i} \text {, }
\end{aligned}
$$


After expressing the terms

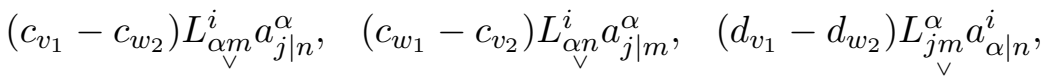

$$
\begin{aligned}
& \left(d_{w_{1}}-d_{v_{2}}\right) L_{j^{\prime n}}^{\alpha} a_{\alpha \mid m}^{i}, \quad\left(d_{w_{1}}+d_{w_{2}}\right) L_{m_{\vee}}^{\alpha} a_{j \mid \alpha}^{i},
\end{aligned}
$$

with respect to the equalities (3.15) 3.19) and substituting them into the equation (1.16), one confirms the validity of the equation (3.33).

The next equalities are satisfied

$$
\begin{aligned}
& \rho_{1}^{z}-\rho_{2}^{z}+\rho_{3}^{z}-\rho_{4}^{z}=(-1)^{1-1} \rho_{1}^{z}+(-1)^{2-1} \rho_{2}^{z}+(-1)^{3-1} \rho_{3}^{z}+\rho^{4-1} \rho_{4}^{z}, \\
& \rho_{1}^{z}-\rho_{2}^{z}-\rho_{3}^{z}+\rho_{4}^{z}=(-1)^{\left\lfloor\frac{1}{2}\right\rfloor} \rho_{1}^{z}+(-1)^{\left\lfloor\frac{2}{2}\right\rfloor} \rho_{2}^{z}+(-1)^{\left\lfloor\frac{3}{2}\right\rfloor} \rho_{3}^{z}+(-1)^{\left\lfloor\frac{4}{2}\right\rfloor} \rho_{4}^{z},
\end{aligned}
$$

$z=1, \ldots, 5$, for the floor function $\lfloor x\rfloor$ (the function that takes as input a real number $x$ and gives the greatest integer less than or equal to $x$ as output).

Let be $\left\{n_{1}, n_{2}, n_{3}, n_{4}\right\}=\{1,2,3,4\}$. With respect to the Proposition 2.2, we conclude that it is enough to consider the case of $\rho_{0}^{z}=\rho_{n_{4}}^{z}=0, n_{4} \in\{1,2,3,4\} \backslash\left\{n_{1}, n_{2}, n_{3}\right\}$.

For integers $n_{1}, n_{2}, n_{3}, 1 \leq n_{1}<n_{2}<n_{3} \leq 4$, and with respect to the equations (3.20, 3.21) substituted into the equation (3.33), the next theorem holds.

Theorem $3.2\left(n_{1}-n_{2}-n_{3}\right.$-Second Ricci-Type Identities Theorem). Let be

$$
\begin{aligned}
& \tilde{X}_{j k}^{i}=\rho_{n_{1}}^{1} a_{j \mid k}^{i}+\rho_{n_{2}}^{1} a_{j \mid k}^{i}+\rho_{n_{3}}^{1} a_{j \mid k}^{i}, \quad \tilde{Y}_{n_{3}}^{i}=\rho_{n_{1}}^{2} a_{j \mid k}^{i}+\rho_{n_{1}}^{i} a_{j \mid k}^{i}+\rho_{n_{2}}^{i} a_{n_{3}}^{i} a_{j \mid k}^{i},
\end{aligned}
$$

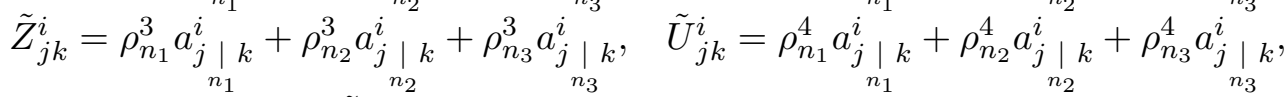

$$
\begin{aligned}
& \tilde{V}_{j k}^{i}=\rho_{n_{1}}^{5} a_{j \mid k}^{i}{ }_{n_{1}}^{i}+\rho_{n_{2}}^{5} a_{j \mid k}^{i}{ }_{n_{2}}^{i}+\rho_{n_{3}}^{5} a_{j \mid k}^{i}{ }_{n_{3}}^{i},
\end{aligned}
$$

for the tensor $\hat{a}$ of the type $(1,1)$.

It holds the equation

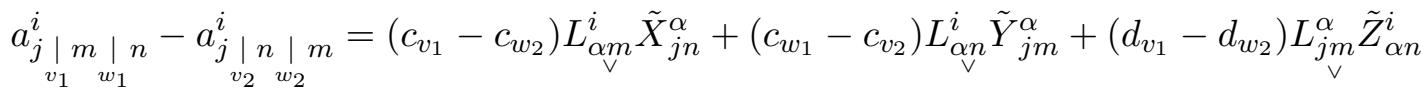

$$
\begin{aligned}
& +\left(d_{w_{1}}-d_{v_{2}}\right) L_{j^{\prime}}^{\alpha} \tilde{U}_{\alpha m}^{i}+\left(d_{w_{1}}+d_{w_{2}}\right) L_{m_{\vee}}^{\alpha} \tilde{V}_{j \alpha}^{i} \\
& +a_{j}^{\alpha}\left\{R_{\alpha m n}^{i}+c_{v_{1}} L_{\alpha \mathrm{V} \mid n}^{i}-c_{v_{2}} L_{\alpha \mathrm{V} \mid m}^{i}\right.
\end{aligned}
$$

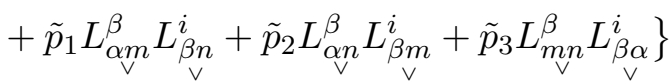

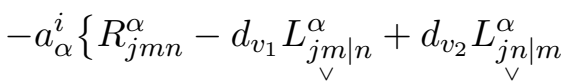

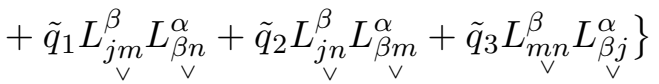

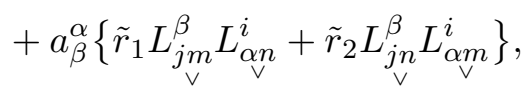


where

$$
\begin{aligned}
& \tilde{p}_{1}=c_{v_{1}} c_{w_{1}}-c_{v_{2}}\left(c_{w_{2}}+d_{w_{2}}\right)-\left(c_{w_{1}}-c_{v_{2}}\right)\left((-1)^{n_{1}-1} \rho_{n_{1}}^{2}+(-1)^{n_{2}-1} \rho_{n_{2}}^{2}+(-1)^{n_{3}-1} \rho_{n_{3}}^{2}\right) \text {, } \\
& \tilde{p}_{2}=c_{v_{1}}\left(c_{w_{1}}+d_{w_{1}}\right)-c_{v_{2}} c_{w_{2}}-\left(c_{v_{1}}-c_{w_{2}}\right)\left((-1)^{n_{1}-1} \rho_{n_{1}}^{1}+(-1)^{n_{2}-1} \rho_{n_{2}}^{1}+(-1)^{n_{3}-1} \rho_{n_{3}}^{1}\right) \text {, } \\
& \tilde{p}_{3}=-c_{v_{1}} d_{w_{1}}-c_{v_{2}} d_{w_{2}}+\left(d_{w_{1}}+d_{w_{2}}\right)\left((-1)^{n_{1}-1} \rho_{n_{1}}^{5}+(-1)^{n_{2}-1} \rho_{n_{2}}^{5}+(-1)^{n_{3}-1} \rho_{n_{3}}^{5}\right) \text {, } \\
& \tilde{q}_{1}=-d_{v_{1}}\left(c_{w_{1}}+d_{w_{1}}\right)+d_{v_{2}} d_{w_{2}}-\left(d_{v_{1}}-d_{w_{2}}\right)\left((-1)^{\left\lfloor\frac{n_{1}}{2}\right\rfloor} \rho_{n_{1}}^{3}+(-1)^{\left\lfloor\frac{n_{2}}{2}\right\rfloor} \rho_{n_{2}}^{3}+(-1)^{\left\lfloor\frac{n_{3}}{2}\right\rfloor} \rho_{n_{3}}^{3}\right) \text {, } \\
& \tilde{q}_{2}=-d_{v_{1}} d_{w_{1}}+d_{v_{2}}\left(c_{w_{2}}+d_{w_{2}}\right)-\left(d_{w_{1}}-d_{v_{2}}\right)\left((-1)^{\left\lfloor\frac{n_{1}}{2}\right\rfloor} \rho_{1}^{4}+(-1)^{\left\lfloor\frac{n_{2}}{2}\right\rfloor} \rho_{n_{2}}^{4}+(-1)^{\left\lfloor\frac{n_{3}}{2}\right\rfloor} \rho_{n_{3}}^{4}\right) \text {, }
\end{aligned}
$$

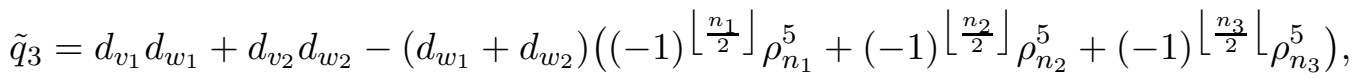

$$
\begin{aligned}
& \tilde{r}_{1}=c_{w_{1}} d_{v_{1}}-c_{v_{2}} d_{w_{2}}+\left(c_{w_{1}}-c_{v_{2}}\right)\left((-1)^{\left\lfloor\frac{n_{1}}{2}\right\rfloor} \rho_{n_{1}}^{2}+(-1)^{\left\lfloor\frac{n_{2}}{2}\right\rfloor} \rho_{n_{2}}^{2}+(-1)^{\left\lfloor\frac{n_{3}}{2}\right\rfloor} \rho_{n_{3}}^{2}\right) \\
& -\left(d_{v_{1}}-d_{w_{2}}\right)\left((-1)^{n_{1}-1} \rho_{n_{1}}^{3}+(-1)^{n_{2}-1} \rho_{n_{2}}^{3}+(-1)^{n_{3}-1} \rho_{n_{3}}^{3}\right), \\
& \tilde{r}_{2}=c_{v_{1}} d_{w_{1}}-c_{w_{2}} d_{v_{2}}+\left(c_{v_{1}}-c_{w_{2}}\right)\left((-1)^{\left\lfloor\frac{n_{1}}{2}\right\rfloor} \rho_{n_{1}}^{1}+(-1)^{\left\lfloor\frac{n_{2}}{2}\right\rfloor} \rho_{n_{2}}^{1}+(-1)^{\left\lfloor\frac{n_{3}}{2}\right\rfloor} \rho_{n_{3}}^{1}\right) \\
& -\left(d_{w_{1}}-d_{v_{2}}\right)\left((-1)^{n_{1}-1} \rho_{n_{1}}^{4}+(-1)^{n_{2}-1} \rho_{n_{2}}^{4}+(-1)^{n_{3}-1} \rho_{n_{3}}^{4}\right), \\
& \rho_{n_{1}}^{z}+\rho_{n_{2}}^{z}+\rho_{n_{3}}^{z}=1, z \in\{1,2,3,4,5\} .
\end{aligned}
$$

With respect to the Proposition 2.2, we conclude that it is enough to consider the case of $\rho_{n_{3}}^{z}=$ $0, \rho_{n_{4}}^{z}=0,1 \leq n_{3}<n_{4} \leq 4,\left\{n_{1}, n_{2}\right\}=\{1,2,3,4\} \backslash\left\{n_{3}, n_{4}\right\},\left(n_{1}, n_{2}\right) \in\{(1,3),(1,4),(2,3),(2,4)\}$ as in the next theorem.

Theorem $3.3\left(n_{1}-n_{2}\right.$-Second Ricci-Type Identities Theorem). Let be

$$
\begin{aligned}
& \tilde{\tilde{X}}_{j k}^{i}=\rho_{0}^{1} a_{j \mid k}^{i}+\rho_{n_{1}}^{1} a_{j \mid k}^{i}+\rho_{n_{1}}^{1} a_{n_{2}}^{i}, \quad \tilde{\tilde{Y}}_{j k}^{i}=\rho_{0}^{2} a_{j \mid k}^{i}+\rho_{n_{1}}^{2} a_{j \backslash k}^{i}+\rho_{n_{1}}^{i} \rho_{n_{2}}^{2} a_{j \mid k}^{i}, \underset{n_{2}}{i}, \\
& \tilde{\tilde{Z}}_{j k}^{i}=\rho_{0}^{3} a_{j \mid k}^{i}+\rho_{n_{1}}^{3} a_{j \mid k}^{i}+\rho_{n_{1}}^{3} a_{j \mid k}^{i}, \quad \tilde{\tilde{U}}_{j k}^{i}=\rho_{0}^{4} a_{j \mid k}^{i}+\rho_{n_{1}}^{4} a_{j \mid k}^{i}{ }_{n_{1}}^{i}+\rho_{n_{2}}^{4} a_{j \mid k}^{i}, \\
& \tilde{\tilde{V}}_{j k}^{i}=\rho_{0}^{5} a_{j \mid k}^{i}+\rho_{n_{1}}^{5} a_{j \mid k}^{i}+\rho_{n_{1}}^{5} a_{j \mid k}^{i},
\end{aligned}
$$

for the tensor $\hat{a}$ of the type $(1,1)$.

It holds the equation 


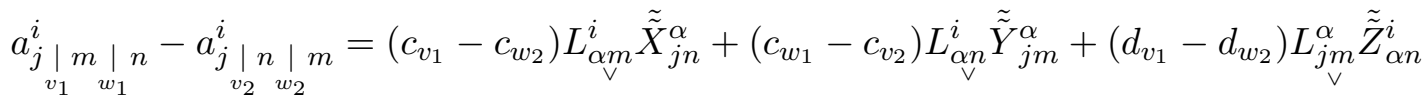

$$
\begin{aligned}
& +\left(d_{w_{1}}-d_{v_{2}}\right) L_{j n}^{\alpha} \tilde{\tilde{U}}_{\alpha m}^{i}+\left(d_{w_{1}}+d_{w_{2}}\right) L_{m_{\vee}}^{\alpha} \tilde{\tilde{V}}_{j \alpha}^{i} \\
& +a_{j}^{\alpha}\left\{R_{\alpha m n}^{i}+c_{v_{1}} L_{\alpha \mathrm{V} \mid n}^{i}-c_{v_{2}} L_{\mathrm{V} \mid m}^{i}\right.
\end{aligned}
$$

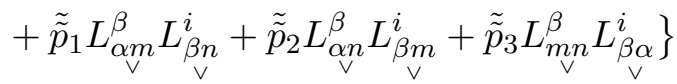

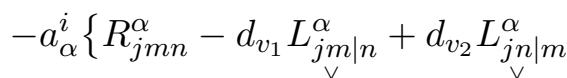

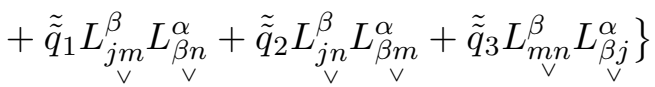

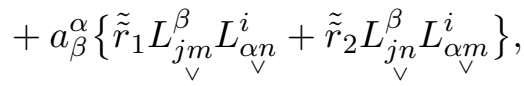

where

$$
\begin{aligned}
& \tilde{\tilde{p}}_{1}=c_{v_{1}} c_{w_{1}}-c_{v_{2}}\left(c_{w_{2}}+d_{w_{2}}\right)-\left(c_{w_{1}}-c_{v_{2}}\right)\left((-1)^{n_{1}-1} \rho_{n_{1}}^{2}+(-1)^{n_{2}-1} \rho_{n_{2}}^{2}\right) \text {, } \\
& \tilde{\tilde{p}}_{2}=c_{v_{1}}\left(c_{w_{1}}+d_{w_{1}}\right)-c_{v_{2}} c_{w_{2}}-\left(c_{v_{1}}-c_{w_{2}}\right)\left((-1)^{n_{1}-1} \rho_{n_{1}}^{1}+(-1)^{n_{2}-1} \rho_{n_{2}}^{1}\right) \text {, } \\
& \tilde{\tilde{p}}_{3}=-c_{v_{1}} d_{w_{1}}-c_{v_{2}} d_{w_{2}}+\left(d_{w_{1}}+d_{w_{2}}\right)\left((-1)^{n_{1}-1} \rho_{n_{1}}^{5}+(-1)^{n_{2}-1} \rho_{n_{2}}^{5}\right) \text {, } \\
& \tilde{\tilde{q}}_{1}=-d_{v_{1}}\left(c_{w_{1}}+d_{w_{1}}\right)+d_{v_{2}} d_{w_{2}}-\left(d_{v_{1}}-d_{w_{2}}\right)\left((-1)^{\left\lfloor\frac{n_{1}}{2}\right\rfloor} \rho_{n_{1}}^{3}+(-1)^{\left\lfloor\frac{n_{2}}{2}\right\rfloor} \rho_{n_{2}}^{3}\right) \text {, } \\
& \tilde{\tilde{q}}_{2}=-d_{v_{1}} d_{w_{1}}-d_{v_{2}}\left(c_{w_{2}}+d_{w_{2}}\right)-\left(d_{w_{1}}-d_{v_{2}}\right)\left((-1)^{\left\lfloor\frac{n_{1}}{2}\right\rfloor} \rho_{n_{1}}^{4}+(-1)^{\left\lfloor\frac{n_{2}}{2}\right\rfloor} \rho_{n_{2}}^{4}\right) \text {, } \\
& \tilde{\tilde{q}}_{3}=d_{v_{1}} d_{w_{1}}+d_{v_{2}} d_{w_{2}}-\left(d_{w_{1}}+d_{w_{2}}\right)\left((-1)^{\left\lfloor\frac{n_{1}}{2}\right\rfloor} \rho_{n_{1}}^{5}+(-1)^{\left\lfloor\frac{n_{2}}{2}\right\rfloor} \rho_{n_{2}}^{5}\right) \text {, } \\
& \tilde{\tilde{r}}_{1}=c_{w_{1}} d_{v_{1}}-c_{v_{2}} d_{w_{2}}+\left(c_{w_{1}}-c_{v_{2}}\right)\left((-1)^{\left\lfloor\frac{n_{1}}{2}\right\rfloor} \rho_{n_{1}}^{2}+(-1)^{\left\lfloor\frac{n_{2}}{2}\right\rfloor} \rho_{n_{2}}^{2}\right) \\
& -\left(d_{v_{1}}-d_{w_{2}}\right)\left((-1)^{n_{1}-1} \rho_{n_{1}}^{3}+(-1)^{n_{2}-1} \rho_{n_{2}}^{3}\right) \text {, } \\
& \tilde{\tilde{r}}_{2}=c_{v_{1}} d_{w_{1}}-c_{w_{2}} d_{v_{2}}+\left(c_{v_{1}}-c_{w_{2}}\right)\left((-1)^{\left\lfloor\frac{n_{1}}{2}\right\rfloor} \rho_{n_{1}}^{1}+(-1)^{\left\lfloor\frac{n_{2}}{2}\right\rfloor} \rho_{n_{2}}^{1}\right) \\
& -\left(d_{w_{1}}-d_{v_{2}}\right)\left((-1)^{n_{1}-1} \rho_{n_{1}}^{4}+(-1)^{n_{2}-1} \rho_{n_{2}}^{4}\right) \text {, }
\end{aligned}
$$

Theorem 3.4 (Commutation Formulae Theorem). Fifteen of the geometrical objects $a_{j|m|}^{i} \underset{v_{1} \mid n}{i}$ -

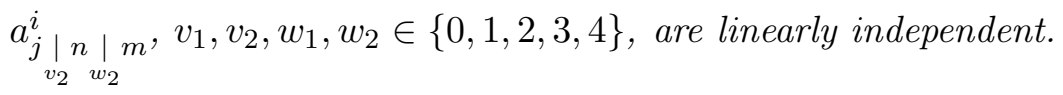

Proof. With respect to the Corollary 2.2 and the equation $(2.6)$ in this corollary, we get

$$
\begin{aligned}
& a_{j \mid v_{1} m}^{i}=x_{1} a_{j \mid m}^{i}+x_{2} a_{j \mid m}^{i}+x_{3} a_{\substack{j \mid m \\
i}}^{i},
\end{aligned}
$$

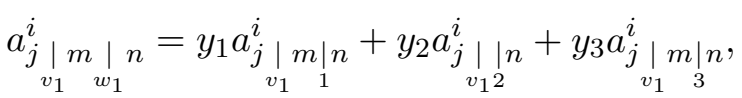

for the corresponding scalars $x_{1}, x_{2}, x_{3}, y_{1}, y_{2}, y_{3}$. 
After substituting the expression (3.42) into the equation (3.43), one gets that the double covariant derivative $a_{v_{1}|m| w_{1}}^{i}$ is a linear combination of the geometrical objects $a_{j|m| n}^{i}{ }_{v_{1}^{\prime} w_{1}^{\prime}}^{i}$, for $v_{1}^{\prime}, w_{1}^{\prime} \in\{1,2,3\}$.

In $1-2-3$-Commutation Formulae Theorem [14], it is proved that sixteen of the geometrical objects $a_{j|m| \underset{v_{1}^{\prime}}{i} w_{1}^{\prime} n}-a_{j|n|}^{i} \underset{v_{2}^{\prime} w_{2}^{\prime}}{i}$, for $v_{1}^{\prime}, v_{2}^{\prime}, w_{1}^{\prime}, w_{2}^{\prime} \in\{1,2,3\}$, are linearly independent, which completes the proof for this theorem.

\section{Identities of Ricci Type with respect to tensor $\hat{a}$ of type $(p, q)$}

It holds the next equation.

$$
\begin{aligned}
& a_{j_{1} \ldots j_{q}|m| n}^{i_{1} \ldots i_{p}}=a_{j_{1} \ldots j_{q}|m| n}^{i_{1} \ldots i_{p}}+c_{v} \sum_{k=1}^{p} L_{\alpha m}^{i_{k}} a_{j_{1} \ldots j_{q} \mid n}^{i_{1} \ldots i_{k-1} \alpha i_{k+1} \ldots i_{p}}+c_{w} \sum_{k=1}^{p} L_{\alpha_{n}}^{i_{k}} a_{j_{1} \ldots j_{q} \mid m}^{i_{1} \ldots i_{k-1} \alpha i_{k+1} \ldots i_{p}} \\
& +d_{v} \sum_{l=1}^{q} L_{j_{l} m}^{\alpha} a_{j_{1} \ldots j_{l-1} \alpha j_{l+1} \ldots j_{q} \mid n}^{i_{1} \ldots i_{p}}+d_{w} \sum_{l=1}^{q} L_{j_{l} n}^{\alpha} a_{j_{1} \ldots j_{l-1} \alpha j_{l+1} \ldots j_{q} \mid m}^{i_{1} \ldots i_{p}}+d_{w} L_{m \eta}^{\alpha} a_{j_{1} \ldots j_{q} \mid \alpha}^{i_{1} \ldots i_{p}}
\end{aligned}
$$

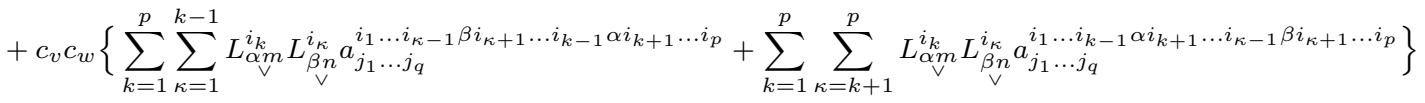

$$
\begin{aligned}
& +d_{v} d_{w}\left\{\sum_{l=1}^{q} \sum_{\ell=1}^{s-1} L_{j_{\ell} n}^{\beta} L_{j_{l} m}^{\alpha} a_{j_{1} \ldots j_{\ell-1} \beta j_{\ell+1} \ldots j_{l-1} \alpha j_{l+1} \ldots j_{q}}^{i_{1} \ldots i_{p}}+\sum_{l=1}^{q} \sum_{\ell=l+1}^{q} L_{j_{\ell} n}^{\beta} L_{j_{l} m}^{\alpha} a_{j_{1} \ldots j_{l-1} \alpha j_{l+1} \ldots j_{\ell-1} \beta j_{\ell+1} \ldots j_{q}}^{i_{1} \ldots i_{p}}\right\}
\end{aligned}
$$

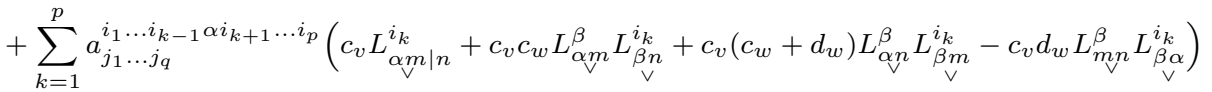

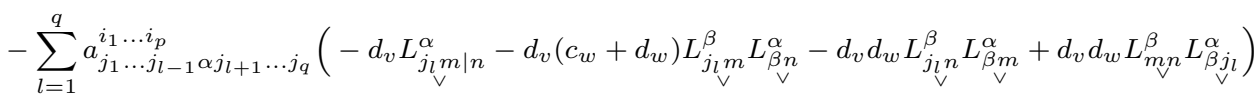

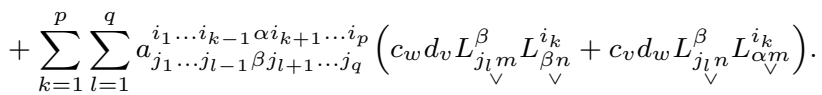

With respect to the equation (2.5), one generalizes the results obtained in the previous section with the next theorems.

Theorem 4.1 (General First Ricci-Type Identities Theorem). The family of identities of the Ricci Type with respect to a non-symmetric affine connection $\nabla$ and a tensor $\hat{a}$ of the type $(p, q)$, $p, q \in \mathbb{N}$, is

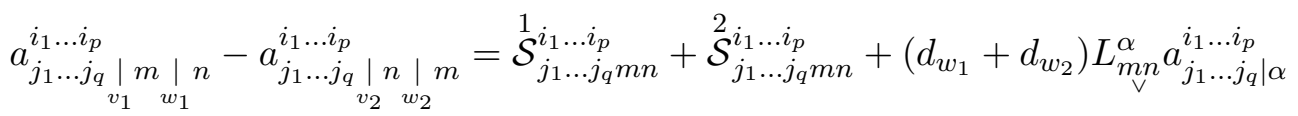

$$
\begin{aligned}
& +\sum_{k=1}^{p} a_{j_{1} \ldots j_{q}}^{i_{1} \ldots i_{k-1} \alpha i_{k+1} \ldots i_{p}} \stackrel{1}{\mathcal{R}}_{\alpha m n}^{i_{k}}-\sum_{l=1}^{q} a_{j_{1} \ldots j_{l-1} \alpha j_{l+1} \ldots j_{q}}^{i_{1} \ldots i_{p}} \stackrel{2}{\mathcal{R}_{j_{l} m n}^{\alpha}} \\
& +\sum_{k=1}^{p} \sum_{l=1}^{q} a_{j_{1} \ldots j_{l-1} \beta j_{l+1} \ldots j_{q}}^{i_{1} \ldots i_{k-1} \alpha i_{k+1} \ldots i_{p}} \stackrel{3}{\mathcal{R}}_{\alpha j_{l} m n}^{\beta i_{k}}+\mathcal{Z}_{j m n}^{i},
\end{aligned}
$$

where 


$$
\begin{aligned}
& \stackrel{1}{\mathcal{S}}_{j_{1} \ldots j_{q} m n}^{i_{1} \ldots i_{p}}=\sum_{k=1}^{p}\left\{\left(c_{v_{1}}-c_{w_{2}}\right) L_{\underset{\vee}{ } i_{m}}^{i_{k}} a_{j_{1} \ldots j_{q} \mid n}^{i_{1} \ldots i_{k-1} \alpha i_{k+1} \ldots i_{p}}+\left(c_{w_{1}}-c_{v_{2}}\right) L_{\substack{\alpha n \\
j_{1} \ldots j_{q} \mid m}}^{i_{k}} a^{i_{1} \ldots i_{k-1} \alpha i_{k+1} \ldots i_{p}}\right\}, \\
& \stackrel{2}{\mathcal{S}}_{j_{1} \ldots j_{q} m n}^{i_{1} \ldots i_{p}}=\sum_{l=1}^{q}\left\{\left(d_{v_{1}}-d_{w_{2}}\right) L_{j_{l} m}^{\alpha} a_{j_{1} \ldots j_{l-1} \alpha j_{l+1} \ldots j_{q} \mid n}^{i_{1} \ldots i_{p}}+\left(d_{w_{1}}-d_{v_{2}}\right) L_{j_{l} n}^{\alpha} a_{j_{1} \ldots j_{r-1} \alpha j_{r+1} \ldots j_{q} \mid m}^{i_{1} \ldots i_{p}}\right\},
\end{aligned}
$$

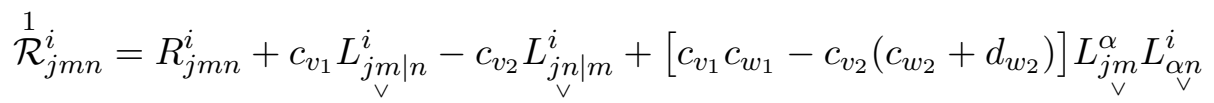

$$
\begin{aligned}
& +\left[c_{v_{1}}\left(c_{w_{1}}+d_{w_{1}}\right)-c_{v_{2}} c_{w_{2}}\right] \underset{\vee}{L_{j n}^{\alpha}} L_{\alpha m}^{i}-\left(c_{v_{1}} d_{w_{1}}+c_{v_{2}} d_{w_{2}}\right) L_{\substack{m_{n} \\
\alpha}}^{\alpha} L_{\vee j}^{i},
\end{aligned}
$$

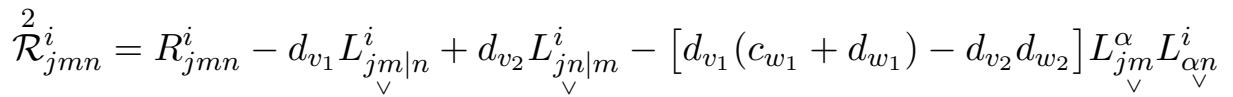

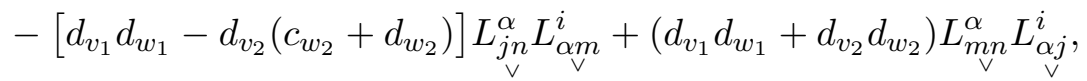

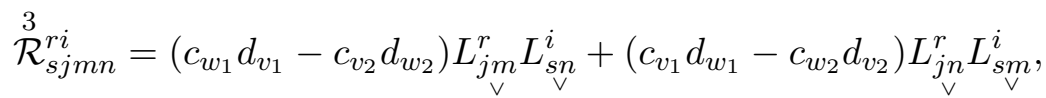

$Z_{j m n}^{i}$ is the sum of the third and the fourth row in the equation (4.1) and $v_{1}, v_{2}, w_{1}, w_{2} \in$ $\{0,1,2,3,4\}$.

\section{Conclusion}

In this article, we generalized the First Ricci-Type Identities Theorem. It was proved that three of geometrical objects $a_{j \mid k}^{i}, a_{j \mid k}^{i}, a_{j \mid k}^{i}, a_{j \mid k}^{i}, a_{j \mid k}^{i}$ are linearly independent here.

After that, we generalized the First Ricci-Type Identities Theorem with respect to a tensor $\hat{a}$ of the type $(p, q), p, q \in \mathbb{N}$.

In the future work, we will generalize the Commutation Formulae Theorem, $n_{1}-n_{2}-n_{3^{-}}$ Second Ricci-Type Identities Theorem and the $n_{1}-n_{2}$-Second Ricci-Type Identities Theorem with respect to tensors of the types $(p, q),(p, 0),(0, q)$.

\section{Acknowledgements}

This research is financially supported by Serbian Ministry of Education, Science and Technological Developments.

The authors thank to the anonymous referee who estimated this paper.

\section{References}

[1] L. P. Eisenhart, Non-Riemannian Geometry, New York, 1927.

[2] J. Mikeš, E. Stepanova, A. Vanžurova, et al.: Differential geometry of special mappings, Olomouc: Palacky University, 2015.

[3] S. M. Minčić, Ricci identities in the space of non-symmetric affine connexion, Mat. Vesnik, 10 (25) sv. 2, (1973), 161-172. 
[4] S. M. Minčić: Curvature tensors of the space of non-symmetric affine connexion, obtained from the curvature pseudotensors, Matematički Vesnik, 13 (28) (1976), 421-435.

[5] S. M. Minčić, New commutation formulas in the non-symmetric affine connexion space, Publ. Inst. Math., Nouv. Sér. 22 (1977) 189-199.

[6] S. M. Minčić, Independent curvature tensors and pseudotensors of spaces with nonsymmetric affine connexion, Coll. Math. Soc. János Bolayai, 31. Dif. geom., Budapest (Hungary), (1979), 445-460.

[7] S. M. Minčić: On Ricci Type Identities in Manifolds With Non-Symmetric Affine Connection, Publications De L'Institut Mathématique, Nouvelle série, tome 94 (108) (2013), 205-217.

[8] S. M. Minčić and Lj. S. Velimirović: Spaces With Non-Symmetric Affine Connection, Novi Sad J. Math., Vol. 38, No. 3, 2008, 157-164.

[9] M. Z. Petrović, Generalized para-Kähler Spaces in Eisenharts Sense Admitting a Holomorphically Projective Mapping, Filomat, Vol. 33, No. 13, 2019, 4001-4012.

[10] M. Z. Petrović, Lj. S. Velimirović, Generalized Kähler spaces in Eisenhart's sense admitting a holomorphically projective mapping, Mediterr. J. Math. (2018) 15:150.

[11] M. Z. Petrović, Lj. S. Velimirović, A new type of generalized para-Kahler spaces and holomorphically projective transformations, Bull. Iran. Math. Soc., Vol. 45, No. 4, 2019, 1021-1043.

[12] N. S. Sinyukov, Geodesic mappings of Riemannian spaces, (in Russian), "Nauka", Moscow, 1979.

[13] M. S. Stanković, M. Lj. Zlatanović, Lj. S. Velimirović, Equitorsion holomorphically projective mappings of generalized Kählerian space of the first kind, Czechoslovak Mathematical Journal, Vol. 60, (2010) 635-653.

[14] N. O. Vesić, Ricci-Type Identities, submitted.

[15] M. Lj. Zlatanović, New projective tensors for equitorsion geodesic mappings, Applied Mathematics Letters, Vol. 25, No. 5, 2012, 890-897. 